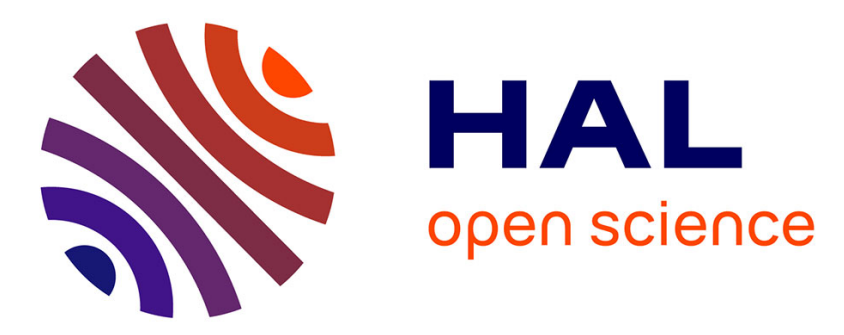

\title{
L'enquête Information et vie quotidienne 2011 parmi les dispositifs nationaux et internationaux de mesure des compétences des adultes. Introduction
}

\author{
Louis-André Vallet
}

\section{- To cite this version: \\ Louis-André Vallet. L'enquête Information et vie quotidienne 2011 parmi les dispositifs nationaux et internationaux de mesure des compétences des adultes. Introduction. Economie et Statistique / Economics and Statistics, 2016, 490, pp.5-15. hal-01482442}

HAL Id: hal-01482442

https://hal-sciencespo.archives-ouvertes.fr/hal-01482442

Submitted on 3 Mar 2017

HAL is a multi-disciplinary open access archive for the deposit and dissemination of scientific research documents, whether they are published or not. The documents may come from teaching and research institutions in France or abroad, or from public or private research centers.
L'archive ouverte pluridisciplinaire HAL, est destinée au dépôt et à la diffusion de documents scientifiques de niveau recherche, publiés ou non, émanant des établissements d'enseignement et de recherche français ou étrangers, des laboratoires publics ou privés.

\section{(ㅇ)(1) $\$$}

Distributed under a Creative Commons Attribution - NonCommercial - NoDerivatives| 4.0 


\title{
L'enquête Information et vie quotidienne 2011 parmi les dispositifs nationaux et internationaux de mesure des compétences des adultes
}

\author{
Louis-André Vallet *
}

Rappel :

Les jugements et opinions exprimés par les auteurs n'engagent qu'eux mêmes, et non les institutions auxquelles ils appartiennent, ni a fortiori l'Insee.
C onduite au sein d'un système éducatif national ou bien sur un ensemble de pays et dans une perspective comparative, la réalisation d'évaluations visant à mesurer les acquis et compétences des élèves d'un âge donné ou d'un niveau scolaire donné est une pratique bien établie depuis plusieurs décennies. Menées sur des populations exhaustives ou des échantillons de grande taille, ces évaluations ont tantôt une visée diagnostique pour permettre aux enseignants de mieux s'adapter aux connaissances de leurs élèves, tantôt une visée de bilan lorsqu'il s'agit de mesurer leurs acquis, l'évolution de ceux-ci et, au-delà, l'efficacité du système éducatif (Trosseille et Rocher, 2015). En France par exemple, de tels dispositifs ont été mis en œuvre par le ministère de l'Éducation nationale à partir de 1975, à l'école élémentaire et au collège, pour couvrir progressivement l'ensemble des disciplines et les différents niveaux scolaires. Puis, à partir de 1989, sont apparues les premières évaluations diagnostiques de masse, réalisées en français et en mathématiques auprès de tous les élèves de cours élémentaire deuxième année et de sixième. Sur le plan international, l'effort de la communauté académique dans cette direction a même été remarquablement précoce. La première enquête de l'International Association for the Evaluation of Education Achievement a en effet été conduite dans douze pays, dès 1960, auprès d'échantillons d'élèves de 13 ans : connue sous le nom de Pilot Twelve-Country Study, elle portait notamment sur les mathématiques, la compréhension de l'écrit, la géographie et les sciences et elle avait précisément pour but de tester la faisabilité de telles opérations extensives, comprenant des tests et questionnaires communs aux différents pays et capables de «fonctionner » dans des contextes culturels différents. Plus récemment, depuis l'année 2000, l'enquête Pisa-Programme international pour le suivi des acquis des élèves - évalue, tous les trois ans, des échantillons d'élèves de 15 ans en lecture/compréhension de l'écrit, mathématiques et sciences, au sein de tous les pays de l'OCDE et de nombreux pays partenaires. Au fil des vagues d'interrogation, ses résultats sont de plus en plus attendus et commentés.

Si la réalisation d'évaluations standardisées auprès de jeunes d'âge scolaire est commune et profite de la facilité d'interrogation au sein des établissements, l'évaluation standardisée des compétences des adultes est un domaine moins naturel et d'un abord plus malaisé. En effet, contrairement au cas des jeunes d'âge scolaire, la légitimité et la finalité d'une évaluation des compétences des adultes ne sont pas d'emblée acquises.

* Directeur de recherche CNRS à Sciences Po, Observatoire sociologique du changement (CNRS), Paris. 
En outre, à supposer que cette première difficulté ait été franchie, plusieurs obstacles d'ordre pratique restent à affronter pour la mise en œuvre. D'une part, des adultes pourraient être moins bien disposés que des élèves à entrer dans une logique d'évaluation. D'autre part, le matériel sur lequel l'évaluation standardisée des compétences s'appuie doit être pertinent pour des adultes appartenant à une tranche d'âge large, de l'ordre de 20 à 65 ans, c'est-à-dire des individus qui appartiennent à un spectre étendu de générations et ont ainsi connu des états très différents du système éducatif et des conditions de scolarisation variées; du fait des écarts d'âge, les mêmes individus se trouvent de plus inégalement éloignés de leur période de formation et ont pu éventuellement garder un mauvais souvenir de celle-ci. Enfin, contrairement au cas des jeunes d'âge scolaire, il n'existe pas de lieu «naturel » dans lequel l'évaluation standardisée des compétences des adultes est susceptible d'être réalisée. Mesure et collecte doivent, le plus souvent, être effectuées au domicile des individus, ce qui engendre des coûts supplémentaires, en argent et en temps, du fait de la dispersion de l'échantillon. Cela soulève aussi des difficultés d'ordre pratique, liées à l'immersion dans le domaine privé - par exemple le risque d'une évaluation des compétences de moindre qualité si l'adulte interrogé est dérangé, ou bien si un autre membre du ménage interfère, etc. L'existence de ces défis explique probablement que l'évaluation standardisée des compétences des adultes se soit développée plus tardivement que celle des jeunes d'âge scolaire.

\section{Une brève histoire des dispositifs d'enquête pour l'évaluation standardisée des compétences des adultes}

Les finalités d'une évaluation sur population adulte mettent généralement l'accent sur l'importance de la lecture et du calcul dans la vie personnelle et professionnelle des individus, c'est-à-dire sur l'appréhension de leurs compétences en « littératie » et en « numératie ", pour reprendre le vocabulaire couramment utilisé sur le plan international. La préoccupation sous-jacente consiste alors à savoir si les habitants d'un pays témoignent d'un degré de maîtrise suffisant dans ces deux dimensions pour leur permettre de faire face aux mutations de la vie quotidienne et des univers professionnels, lesquelles les confrontent à une information de plus en plus volumineuse, diverse et complexe (Murat et Rocher, 2015). En particulier, l'évaluation des compétences des adultes actifs renvoie à celle de l'efficacité de la formation tout au long de la vie - en milieu professionnel pour ceux qui ont un emploi ou proposée aux demandeurs d'emploi - et aux questions d'inadéquation entre compétences et emplois (skill mismatch). Au-delà de cette préoccupation, ces dispositifs d'évaluation permettent de décrire le niveau de compétence de l'ensemble de la population adulte du pays, d'étudier comment elle se répartit dans les différents niveaux qui ont pu être identifiés. Ils autorisent aussi une meilleure connaissance de la population des personnes les plus en difficultés face à l'écrit - une situation désignée en France sous le terme d'illettrisme.

\section{D'abord à l'international...}

C'est en 1985 et aux États-Unis qu'a été réalisée la première enquête sur grand échantillon pour mesurer les compétences en littératie des adultes. Organisée par Educational Testing Service (ETS) avec le soutien du National Center for Educational Statistics (NCES), la Young Adult Literacy Survey (YALS) ne concernait en réalité que les jeunes adultes, mais elle a permis d'expérimenter un dispositif d'évaluation constitué d'épreuves utilisant des 
supports diversifiés et présentant des niveaux de difficulté divers. Puis, en 1989-1990, la National Adult Literacy Survey $(N A L S)$ a porté sur un échantillon représentatif de toute la population adulte des États-Unis. Elle visait à appréhender trois dimensions : la capacité à comprendre et à utiliser l'information contenue dans des textes présentés sur des supports variés (prose literacy); l'aptitude à manipuler de l'information contenue dans des tableaux (document literacy) ; enfin, le degré d'aisance dans l'usage de l'arithmétique pour des situations de la vie quotidienne (quantitative literacy) ${ }^{1}$.

Ensuite et sur le plan international, l'enquête International Adult Literacy Survey (IALS) a été organisée par Statistique Canada et ETS, puis coordonnée par l'OCDE, entre 1994 et 1998, dans une vingtaine de pays. Elle a donné lieu à des études comparatives (OCDE et Statistique Canada, 2000) qui analysaient les niveaux de compétences observés dans les pays participants. Pour ce faire et sur la base des items de l'enquête traduits dans les différentes langues, une échelle internationale commune a été construite, à partir d'une approche fondée sur les modèles de réponse à l'item $(\mathrm{MRI})^{2}$ et fréquemment utilisée en psychométrie. Dès 1994, la France a participé à l'enquête $I A L S$, mais les résultats la concernant ne figurent pas dans la publication finale de l'étude car elle a souhaité quitter ce dispositif, jugeant ces résultats peu vraisemblables : $40 \%$ des adultes français étaient classés dans le niveau de compétences le plus faible, caractéristique des plus mauvais lecteurs, et cette proportion excédait de beaucoup celle observée dans la plupart des pays participants, par exemple l'Allemagne, la Belgique, le Royaume-Uni ou la Suisse - voir plus loin l'analyse de ces résultats peu plausibles.

En 1999, l'OCDE, avec Statistique Canada et ETS, a lancé une nouvelle enquête sur les compétences des adultes - l'Adult Literacy and Life Skills survey (ALL) - qui a ajouté aux dimensions de littératie et numératie, déjà présentes dans $I A L S$, celle de résolution de problèmes (problem solving). Les principes de construction du questionnaire étant inchangés par rapport à l'enquête précédente, la France a pris la décision de ne pas être partie prenante de $A L L S$, mais d'orienter ses efforts vers la conception d'une méthodologie et d'un instrument spécifiques qui ont donné naissance aux enquêtes Information et vie quotidienne conduites en 2004 puis en 2011. D'autres pays, notamment l'Allemagne et le Royaume-Uni, ont aussi pris la décision de ne pas participer à l'enquête $A L L$.

La France a en revanche été partie prenante du programme PIAAC-Programme for the International Assessment of Adult Competencies - qui a été lancé par l'OCDE en 2007 et dont la collecte a été réalisée dans 24 pays entre 2011 et 2012. Les compétences mesurées sont les mêmes que pour l'enquête $A L L$, mais la passation du protocole se déroule désormais sur ordinateur autour d'exercices interactifs à résoudre - sauf dans le cas où l'individu enquêté ne maîtrise pas suffisamment l'outil informatique qui est alors remplacé par un support papier. L'OCDE a diffusé les résultats du programme

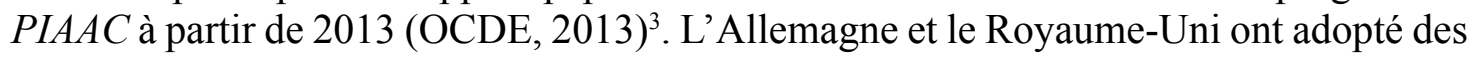
démarches proches de la France, en réalisant une enquête nationale sur les compétences et en participant aussi au programme PIAAC.

\footnotetext{
1. Pour des informations complémentaires sur le développement des enquêtes en littératie et numératie, on pourra consulter l'introduction d'Arnaud Degorre et Fabrice Murat au numéro 424-425 d'Économie et Statistique, publié en 2009 et entièrement consacré à l'enquête Information et vie quotidienne 2004.

2. Ou IRT pour «Item Response Theory » dans l'univers anglo-saxon.

3. Voir aussi le site http://www.oecd.org/skills/piaac/publications.htm pour un aperçu complet des publications et documents de travail de l'OCDE relatifs au programme PIAAC.
} 


\section{... puis en France}

Les résultats, surprenants et peu vraisemblables, obtenus pour la France dans le cadre de l'enquête $I A L S$ ont été à l'origine de travaux d'expertise méthodologique (cf. notamment Blum et Guérin-Pace, 2000 ; Bonnet et al., 2001 ; Dickes et Vrignaud, 1995). Ils ont fait apparaître l'impact possible de la traduction à partir de l'anglais pour certains items, l'inadaptation relative de certains exercices selon le contexte culturel, ou encore l'influence de la codification des réponses - notamment le fait que la non-réponse à une question donnée soit systématiquement assimilée à une réponse fausse. Mais ce sont surtout les conditions de passation du protocole de l'enquête $I A L S$ qui ont été interrogées : on avait remis à chaque individu enquêté un cahier contenant l'ensemble des textes et exercices, le sujet devant alors répondre librement, éventuellement dans l'ordre de son choix. L'épaisseur du cahier, qui laissait entrevoir la longueur probable de la passation, a pu décourager des enquêtés et susciter aussi des réactions défavorables face à un stimulus évoquant trop le cadre scolaire. En outre, l'expertise méthodologique a pu montrer que, à l'intérieur du cahier, les exercices ne se succédaient pas selon un niveau de difficulté croissant, mais qu'ils étaient plutôt présentés dans un ordre aléatoire - un item très difficile pouvant ainsi précéder un item facile, ce qui pouvait alors perturber la personne. On soulignera aussi que les enquêteurs avaient pour consigne d'éviter toute interaction avec les enquêtés et qu'ils jouaient en quelque sorte un rôle de surveillant au cours de l'administration de l'épreuve. Bref, sur ces deux derniers points, l'administration concrète de l'enquête $I A L S$ contrevenait à deux principes habituels en psychométrie : la présentation des items selon un ordre de difficulté croissante, et une attitude bienveillante de la personne administrant l'épreuve à l'égard de celle qui la subit, en vue de soutenir l'attention et la motivation de cette dernière, tout au long de la passation. Enfin, l'expertise méthodologique a permis d'établir qu'il avait été fréquent que les enquêtés répondent en fonction de leurs propres connaissances, et non pas à partir des informations incluses dans le texte fourni, voire qu'ils répondent en critiquant l'éventail des solutions proposées. Dans ce dernier cas, leur réponse avait donc été comptabilisée comme fausse, alors même que la critique exprimée établissait clairement qu'ils avaient bien compris le texte.

À la suite de l'identification de ces difficultés, un comité de pilotage a été constitué en vue de mettre en place un protocole plus satisfaisant pour la mesure des compétences des adultes dans le cadre d'une enquête de la statistique publique auprès des ménages ${ }^{4}$. Un regroupement pluridisciplinaire mêlant notamment des statisticiens d'enquête, des spécialistes de psychométrie et des chercheurs en sciences sociales, a été épaulé par des équipes de recherche universitaires en psychologie ${ }^{5}$ qui ont travaillé à la confection des exercices et à leur adaptation aux contraintes engendrées par la situation d'enquête à domicile. La réalisation de deux enquêtes-pilotes, en 2000 et 2002, a démontré la faisabilité d'une mesure des compétences en littératie et en numératie, grâce à l'utilisation de supports variés - un programme de télévision, un $\mathrm{CD}$, des cartes routières, des textes de longueur et de difficulté diverses, etc., tous supports choisis dans le but d'évoquer le moins possible le contexte scolaire - dans le cadre d'un protocole administré par ordinateur où l'interaction entre enquêteur et enquêté, au cours de la présentation

4. Outre l'Insee, le comité de pilotage regroupait des représentants de l'Agence nationale de lutte contre l'illettrisme (ANLCl), du Centre de recherche en économie et statistique (Crest), de la Direction de l'animation de la recherche, des études et des statistiques (Dares, ministère du Travail), de la Direction de l'évaluation, de la prospective et de la performance (Depp, ministère de l'Éducation nationale), de l'Institut national d'études démographiques (Ined) et de l'Institut national d'étude du travail et d'orientation professionnelle (Inetop). 5. II s'agit des équipes PsyEF (Université Lyon II) sur le module d'orientation et le module "ANLCI ", EVA (Université Rennes II et Hôpital Sainte-Anne) sur le module d'orientation et le module "Numératie », Paris V et Paris XIII sur le module « Haut ». 
successive des exercices, permet de soutenir l'attention et la motivation de ce dernier (Vallet et al., 2002).

Après ces essais qui l'ont très largement inspirée, l'enquête Information et vie quotidienne 2004 - qui a bénéficié du label d'intérêt général et de qualité statistique assorti du caractère obligatoire - a été conduite dans l'ensemble des régions de France métropolitaine, à partir d'un échantillon de 17300 logements et en surreprésentant les ménages avec les probabilités les plus fortes d'y trouver une personne en difficulté face à l'écrit. On a fait en sorte de pouvoir disposer de résultats relatifs aux zones urbaines sensibles (ZUS). Des extensions régionales ont aussi été réalisées en Aquitaine, Nord - Pas-de-Calais et Pays de la Loire pour y disposer de résultats représentatifs. Enfin, après certaines adaptations visant à tenir compte du contexte, le protocole d'IVQ 2004 a été administré en Martinique (2006), à la Réunion (2007) et en Guadeloupe (2008).

L'enquête Information et vie quotidienne 2011 - qui fait l'objet du présent numéro constitue, avec quelques aménagements, une nouvelle édition d'IVQ 2004. Elle a été organisée par l'Insee en collaboration avec l'ANLCI, la Dares et la Depp, ainsi que le Département des statistiques, des études et de la documentation (DSED, ministère de l'Intérieur), la Délégation générale à la langue française et aux langues de France (DGLFLF, ministère de la Culture) et le Secrétariat général du comité interministériel des villes (SG-CIV). Conduite en France métropolitaine avec, de nouveau, des extensions régionales en Haute-Normandie, Île-de-France, Nord - Pas-de-Calais, Picardie et Provence-Alpes-Côte d'Azur, IVQ 2011 a concerné 14000 personnes et permet d'établir des comparaisons avec l'enquête réalisée sept ans plus tôt ${ }^{6}$.

\section{Information et vie quotidienne de 2004 à 2011}

L'architecture d'ensemble des enquêtes $I V Q$ a été précisément décrite dans l'introduction au numéro consacré à l'édition de $2004^{7}$ et on la résumera donc ici succinctement. Il s'agit, à la fois, "de disposer d'éléments d'évaluation communs à l'ensemble de la population enquêtée, et d'adapter au mieux le questionnaire au niveau des compétences de l'enquêté, tel qu'il est mesuré au fur et à mesure des items » (Degorre et Murat, 2009, 13-14).

Pour ce faire, après la désignation par tirage au hasard du membre du ménage qui sera interrogé $e^{8}$, un module dit «d'orientation » lui est proposé. À partir de la présentation d'une page d'un programme de télévision, des exercices d'identification de mots, de compréhension et de calcul permettent d'évaluer la capacité à lire des mots isolés et à comprendre un texte simple.

Dans le cas où l'enquêté ne commet pas ou commet peu d'erreurs, il est orienté vers un module dit «Haut», composé d'exercices portant sur des supports variés - textes ou graphiques - et qui permettent d'évaluer le degré de maîtrise de compétences plus élaborées - par exemple, la capacité à construire des inférences.

6. Information et vie quotidienne a aussi été conduite en Guyane et à la Réunion (2011-2012), à Mayotte et en Nouvelle-Calédonie (2012-2013), ainsi qu'en Martinique (2014).

7. Degorre et Murat, 2009, 13-23.

8. Parfois délicat à imposer au ménage, le tirage aléatoire du répondant est pourtant une condition sine qua non de la validité des résultats de l'enquête. Il évite en particulier le biais qui pourrait être introduit si les membres du ménage eux-mêmes désignaient parmi eux la personne qu'ils considèrent spontanément comme « la plus compétente » pour répondre. 
Lorsque les résultats à l'exercice d'orientation sont faibles, l'enquêté est dirigé vers un module dit "ANLCI » qui permet de mieux identifier les difficultés qu'il rencontre. Outre des questions sur la lecture de mots et la compréhension de textes simples - à partir d'un CD de musique, les exercices de ce module évaluent la capacité à produire des mots écrits - à partir de la « dictée » d'une liste de courses. La performance minimale des enquêtés dans ces trois domaines fondamentaux permet alors d'établir une différenciation selon le degré de difficulté face à l'écrit - difficultés partielles, difficultés fortes ou graves difficultés.

Enfin, lorsque la performance de l'enquêté à l'exercice d'orientation est de niveau intermédiaire, il lui est proposé l'exercice de compréhension sur CD du module ANLCI puis, selon les résultats à celui-ci, la redirection se fait, soit vers le module « Haut», soit vers le restant du module ANLCI.

Par ailleurs, toutes les personnes enquêtées sont soumises à un exercice de compréhension orale et à de courts problèmes de calcul posés par l'enquêteur. L'interrogation s'achève par le recueil d'un ensemble d'éléments biographiques sur l'enfance, la scolarité et l'histoire professionnelle de l'enquêté. Tout au long de l'administration du protocole, des métadonnées sont recueillies, soit par ordinateur pour mesurer les temps de réponse à un exercice, soit directement par l'enquêteur au moyen d'une grille d'observation relative au degré de motivation ou de lassitude de l'enquêté ainsi qu'aux événements perturbateurs éventuels.

Dans cette architecture générale inchangée, deux modifications ont été introduites lors de l'enquête de 2011. D'une part, le module " Haut» a été enrichi en vue de mieux décrire les compétences des individus qui y sont soumis ; l'analyse de l'enquête de 2004 avait en effet montré qu'elle était insuffisamment discriminante dans le haut de la distribution. D'autre part, un module commun a été proposé à tous en fin d'évaluation ; il permet notamment de construire, de manière suffisamment robuste, un score commun sur l'ensemble de la population observée. Ces deux transformations ne remettent pas en cause la possibilité d'une comparaison entre les deux éditions de l'enquête $I V Q^{9}$; elles ont néanmoins occasionné un travail méthodologique conséquent pour y parvenir ${ }^{10}$.

Les premiers résultats ont fait apparaître que, en 2011, $16 \%$ des personnes de 18 à 65 ans résidant en France métropolitaine éprouvent des difficultés dans l'un au moins des trois domaines fondamentaux de l'écrit ${ }^{11}$, ces difficultés étant évaluées comme graves ou fortes pour $11 \%$ (Jonas, 2012). Si l'on considère les seuls individus qui ont été scolarisés en France, $7 \%$ sont dans cette dernière situation et peuvent donc être considérés comme en situation d'illettrisme - soit environ 2,5 millions de personnes de la tranche d'âge retenue. La proportion correspondante était évaluée à $9 \%$ en 2004 . Le recul de deux points du taux d'illettrisme s'expliquerait par un effet de génération. D'une part, les personnes nées avant 1946 - pour lesquelles l'accès à l'enseignement secondaire a été peu fréquent - sont exclues du champ de l'enquête de 2011 alors qu'en 2004, près d'un quart d'entre elles étaient en difficultés graves ou fortes face à l'écrit. D'autre part, les jeunes nés après 1986 n'étaient pas présents en 2004, mais ils le sont en 2011 et, parmi eux, le taux de difficultés graves ou fortes s'élève à $5 \%$.

9. Certaines modifications, plus mineures, ont aussi été introduites. Par exemple, le programme de télévision présenté en début d'enquête a été adapté pour tenir compte des évolutions intervenues.

10. Voir l'article de Murat et Rocher dans ce numéro.

11. C'est-à-dire lecture de mots, compréhension de textes simples et production de mots écrits. 
Plus généralement, l'aisance dans la réalisation des exercices varie avec l'âge, au fur et à mesure que s'éloigne la période où l'on a fréquenté le système scolaire. Que l'on considère le domaine de l'écrit, celui du calcul ou encore celui de la compréhension orale, les plus jeunes rencontrent moins fréquemment des difficultés que les plus âgés. Par exemple, en 2011 et en compréhension orale, $11 \%$ seulement des moins de 30 ans ont réussi moins de $60 \%$ des exercices proposés, mais la proportion s'élève à $17 \%$ des 50-59 ans et 24\% des 60-65 ans. On relève encore que, d'un domaine à l'autre, les mauvaises performances ont tendance à se cumuler. Ainsi, près d'une personne sur deux en difficulté à l'écrit l'est également en compréhension orale ou en calcul. Le cumul n'a toutefois pas de caractère systématique : par exemple, $21 \%$ des personnes en difficulté à l'écrit réalisent d'excellentes performances en compréhension orale. Enfin, IVQ 2011 confirme un résultat qui émergeait déjà d'IVQ 2004 comme des enquêtes Pisa auprès des jeunes de 15 ans : à l'exception de la compréhension orale, l'écart entre hommes et femmes est marqué, mais il est de sens opposé à l'écrit et en calcul. Dans ce dernier domaine, près de $20 \%$ des femmes ont des résultats médiocres contre $14 \%$ des hommes. En revanche, la proportion de personnes qui rencontrent des difficultés - partielles, fortes ou graves - dans le domaine de l'écrit est un peu plus forte parmi les hommes $(17 \%)$ que parmi les femmes $(15 \%)$ et cela se vérifie aussi dans chacun des trois domaines fondamentaux de l'écrit.

\section{Entre Information et Vie Quotidienne 2011 et PIAAC 2012 : la sensibilité de la mesure à l'instrument utilisé}

La présente livraison d'Économie et Statistique n'inclut pas d'article consacré à l'enquête $P I A A C$, sur les seules données françaises ou bien en comparaison internationale, ou encore d'article qui exploite à la fois, voire confronte, IVQ 2011 et $P I A A C^{12}$. On dispose cependant de premiers résultats situant la France parmi les 24 pays de l'OCDE participants, du point de vue des capacités des adultes à maîtriser des informations écrites ou chiffrées (Jonas, 2013). Par ailleurs, une autre publication s'est efforcée de comparer les deux enquêtes en vue d'expliquer qu'elles puissent produire des résultats différents (Jonas et al., 2013).

Selon l'enquête PIAAC, en France métropolitaine, 21,6 \% des personnes âgées de 16 à 65 ans ont un faible niveau de compétence en littératie - résultat qui place la France à un rang inférieur à la moyenne des 24 pays participants $(15,5 \%)$, mais néanmoins assez proche de l'Allemagne et des États-Unis (17,5\%), l'Italie et l'Espagne étant les seuls à compter des proportions plus élevées de faible niveau (respectivement $27,7 \%$ et 27,5 \%). De même, en France, pour ce qui concerne la numératie, 28,0 \% des personnes de 16 à 65 ans ont un faible niveau de compétence - résultat ici encore moins bon que la moyenne des pays participants (19,0\%), l'Italie, l'Espagne et les États-Unis étant les seuls à présenter une proportion plus élevée (respectivement 31,7\%,30,6\% et 28,7\%). En littératie / domaine de l'écrit comme en numératie / domaine du calcul et pour des champs très proches, PIAAC fournit donc des taux de population en difficulté systématiquement supérieurs à ceux d'IVQ 2011. Comment rendre compte de cette discordance ?

12. La raison en est probablement que la collecte de PIAAC a été réalisée plus tardivement que celle d'IVQ 2011, mais aussi que les chercheurs français sont vraisemblablement moins familiers de la méthodologie spécifique d'analyse de la première enquête, en particulier le fait de disposer de plusieurs "valeurs plausibles » plutôt que d'un score de performance unique. 
En premier lieu, il convient de souligner que les deux protocoles d'enquête sont assez différents. En effet, s'agissant de PIAAC, chaque individu débute l'interrogation par un questionnaire biographique - parcours scolaire et professionnel, compétences mobilisées dans le travail, actuel ou passé, et hors travail. Puis il passe de façon autonome, sur ordinateur $^{13}$ et en l'absence de toute interaction avec l'enquêteur, à une série d'exercices dont le niveau de difficulté est en partie adaptatif. L'ordonnancement est donc à l'inverse de celui d'IVQ 2011 et l'attention portée au soutien de la motivation de l'enquêté est indubitablement moindre. Par ailleurs, la durée totale de l'enquête s'avère nettement différente : d'un peu plus d'une heure pour $I V Q 2011$, elle peut atteindre jusqu'à deux heures pour PIAAC, le fait que les exercices succèdent au questionnaire biographique - plutôt que le précèdent - pouvant encore accentuer un phénomène de fatigue.

Plus fondamentalement, il est probable que «ce qui est mesuré précisément » diffère quelque peu entre les deux protocoles. À l'origine des enquêtes Information et vie quotidienne se trouve la question de la mesure de l'illettrisme, liée aux thématiques de la pauvreté et de l'exclusion sociale. Le module ANLCI y revêt une importance particulière et c'est en adoptant une approche multidimensionnelle, comprenant des épreuves dans trois domaines - lecture des mots, compréhension écrite et production écrite, qu'il permet d'identifier finement les niveaux les plus faibles de la maîtrise de l'écrit. Pour ce qui concerne PIAAC, l'utilisation de la méthodologie des modèles de réponse à l'item conduit à définir le niveau de compétence comme un trait latent unidimensionnel par rapport auquel il est possible de situer l'ensemble de la population. La définition des groupes de compétence - et notamment du groupe le plus faible - est alors réalisée a posteriori, par découpage au sein de la distribution d'ensemble des scores.

Des travaux complémentaires restent à effectuer qui utiliseraient simultanément les deux enquêtes pour mieux les comparer. Et il faut aussi souligner qu'au-delà de la mesure des compétences des adultes comme de l'identification de leurs déterminants - thèmes traités dans les articles qui suivent ou dans la livraison d'Économie et Statistique relative à IVQ 2004, la question des effets ou conséquences des compétences, au travail ou dans la vie hors travail, reste insuffisamment traitée dans la littérature de langue française ${ }^{14}$.

\section{Perspectives pour les enquêtes sur les compétences des adultes}

Alors que l'enquête $I V Q$ est davantage tournée vers la mesure de l'illettrisme et les difficultés importantes des adultes face à l'écrit et au calcul, et que PIAAC vise à mesurer l'ensemble des niveaux de compétences en littératie et en numératie dans une perspective internationale, des réflexions sont engagées au sein du Système statistique public français, notamment à l'Insee, à la Depp et à la Dares, sur l'organisation la plus efficace afin d'atteindre deux buts : d'une part, continuer à participer à des programmes de comparaison internationale en matière de compétences des adultes, en contribuant à la prochaine vague du programme PIAAC (2021); d'autre part, poursuivre la mesure de l'importance de l'illettrisme en adaptant les différents supports d'enquête à l'évolution des nouvelles technologies.

13. Sauf exception, mais l'administration "sur papier » a été plus fréquente parmi les individus ultérieurement classés dans les niveaux de compétences les plus faibles et, par ailleurs, la question de la stricte équivalence entre les deux formes d'administration - ordinateur et papier - reste posée.

14. Voir cependant le document d'études d'Aline Branche-Seigeot (2015). 


\section{Au sommaire du numéro}

Les articles qui forment le présent numéro approfondissent, sous divers aspects, l'analyse des données de l'enquête Information et vie quotidienne 2011.

Les deux premiers textes mettent en œuvre des méthodes spécifiques d'analyse textuelle et d'analyse du langage associées à des classifications hiérarchiques. Le premier (Baril et Guérin-Pace) cherche à éclairer la relation entre difficultés à l'écrit à l'âge adulte et événements marquants dans l'enfance, éléments qui sont recueillis en complément d'un questionnaire biographique et aussi, nouveauté de l'enquête $I V Q 2011$ par rapport à $I V Q$ 2004, au moyen d'un questionnement ouvert afin de laisser la possibilité aux répondants de s'exprimer sur des sujets sensibles - cas de violence par exemple. L'utilisation de l'analyse textuelle, une méthode de traitement des Big data constituées de mots et de textes, méthode éprouvée depuis une vingtaine d'années dans le domaine des sciences sociales, permet d'explorer le large corpus de mots ainsi rassemblé. L'étude met en évidence que les situations pouvant être qualifiées d'illettrisme ne s'observent qu'au sein d'une seule classe d'individus ( $7 \%$ des répondants scolarisés en France), ayant vécu une enfance difficile avec un parcours scolaire extrêmement réduit dans un environnement familial relativement violent.

Le deuxième (Blondel et al.) se concentre sur la production écrite de mots, épreuve qui prend la forme d'une liste de courses à écrire sous la dictée, et à laquelle sont soumises les personnes repérées en difficulté en lecture ou en compréhension écrite lors de l'enquête. Cette épreuve fait partie du module conçu par l'Agence nationale de lutte contre l'illettrisme (ANLCI) et participe au diagnostic de celui-ci. L'examen de ces productions écrites du point de vue de la correspondance graphophonologique, qui évalue les mots écrits selon leur correspondance avec l'oral indépendamment de l'exactitude orthographique, met en évidence la diversité des profils langagiers des personnes qui ont passé l'épreuve de production écrite. De fortes difficultés à l'écrit sont toutefois un assez bon révélateur des difficultés en compréhension de l'écrit; de plus, une bonne réussite en orthographe ne va pas systématiquement de pair avec une bonne compréhension de l'écrit ou de l'oral.

L'article suivant (Murat et Rocher) cherche à expliciter le constat, à partir d'IVQ et de $P I A A C$, selon lequel les individus les plus âgés ont de moins bons résultats que les plus jeunes tant en compréhension de l'écrit qu'en calcul. Ces moins bonnes performances peuvent s'expliquer par une perte de compétences au fil des ans, notamment du fait d'une moindre utilisation dans le cadre professionnel ou dans la vie quotidienne (effet « cycle de vie »). Mais elles peuvent aussi signifier une amélioration du niveau moyen des générations les plus récentes (effet " génération »). La réédition de l'enquête $I V Q$ en 2011 après 2004 donne l'opportunité de distinguer entre ces deux phénomènes : l'effet de cycle de vie apparaît fort et la perte de compétences s'accroît après 45 ans ; l'effet de génération est de moindre ampleur en faveur des plus jeunes, mais il s'inverse, à âge et diplôme fixés. Ces résultats rejoignent ceux obtenus à partir de l'enquête PIAAC. Dans le contexte du vieillissement de la population, la détérioration des compétences avec l'âge mise en évidence pourrait suggérer des politiques de formation spécifique aux actifs seniors dans le cadre des dispositifs de formation tout au long de la vie. La légère amélioration des compétences d'une génération à l'autre peut être portée au crédit de l'allongement des études et de l'élévation des niveaux de diplôme. Néanmoins, les analyses à niveau de diplôme fixé étayent l'hypothèse d'une certaine dévalorisation des diplômes. 
Ce troisième article met aussi l'accent sur un point méthodologique crucial, qui devra être pris en compte dans les réflexions sur les prochaines éditions des enquêtes $I V Q$ et PIAAC. La légère évolution du protocole d'évaluation entre 2004 et 2011 a entraîné un travail complexe, s'appuyant notamment sur les modèles de réponses à l'item, afin d'assurer la comparabilité entre les deux éditions. La diffusion des nouvelles technologies, ces années récentes, rend indispensable l'évolution de certains supports des enquêtes - par exemple la page de programme de télévision qui ne peut plus passer pour un document universellement utilisé - comme le développement d'exercices sur supports électroniques - smartphones ou tablettes notamment. Les évolutions de protocole consécutives rendront encore plus difficile le travail de calage indispensable à la comparabilité des compétences des adultes au cours du temps.

Le dernier article de ce numéro (Jourdan et al.) porte sur des informations inédites concernant les difficultés à l'écrit et à l'oral des nouveaux migrants - à savoir les personnes originaires de pays tiers à l'Espace économique européen et à la Suisse, qui ont obtenu en 2009 un premier titre de séjour pour une durée d'au moins un an - à partir de l'Enquête longitudinale sur l'intégration des primo-arrivants (Elipa), menée durant les années 2010, 2011 et 2013. À partir du rapprochement de ces informations avec celles de l'enquête $I V Q 2011$, l'étude met en évidence que, comparés aux autres immigrés, en particulier des pays tiers, et aux non-immigrés, les nouveaux migrants ont des performances moins bonnes. De plus, une fois tenu compte de leurs caractéristiques sociodémographiques - moins diplômés, plus fréquemment au foyer, etc. - l'âge de l'apprentissage de la lecture et de l'écriture du français apparaît comme un déterminant important de leurs performances.

L'accélération des flux migratoires depuis le début des années 2000 vers les pays de l'OCDE, et plus encore la crise des réfugiés, ont suscité des recommandations et des évolutions en matière de politiques publiques dirigées vers les nouveaux migrants. Parmi celles-ci figurent des programmes pour l'apprentissage de la langue du pays d'accueil et une insertion rapide dans le marché du travail (OCDE, 2016). Les analyses de cette étude confirment la pertinence de ces politiques et soulignent l'intérêt de disposer de données permettant l'étude de certains aspects de l'intégration des nouveaux migrants.

Les résultats de ces différents articles sont ainsi susceptibles d'éclairer les recommandations de politiques publiques d'éducation et de formation, notamment en faveur de la formation tout au long de la vie, et de les compléter par des politiques ciblées à l'égard de populations spécifiques, que ce soient les adultes ayant vécu une enfance très difficile, les nouveaux migrants ou les actifs seniors.

\section{BIBLIOGRAPHIE}

Blum A. et Guérin-Pace F. (2000), Des lettres et des chiffres. Des tests d'intelligence à l'évaluation du "savoir lire », un siècle de polémiques, Paris, Fayard.

Bonnet G., Braxmeyer N., Horner S., Lappalainen H.-P., Levasseur J., Nardi E., Rémond M., Vrignaud P. et White J. (2001), The Use of National Reading Tests for International Comparisons: Ways of Overcoming Cultural Bias, Paris, MEN-DEP. 
Branche-Seigeot A. (2015), « Compétences individuelles et compétences utilisées en situation de travail. Quels constats ? Quelle valorisation salariale?», Document d'études, n 193, Dares.

Degorre A. et Murat F. (2009), « La mesure des compétences des adultes, un nouvel enjeu pour la statistique publique », Économie et Statistique, n 424-425, pp. 3-30.

Dickes P. et Vrignaud P. (1995), Rapport sur les traitements des données françaises de l'enquête internationale sur la littératie, Rapport pour le ministère de l'Éducation nationale, Paris, MEN.

Jonas N. (2012), « Pour les générations les plus récentes, les difficultés des adultes diminuent à l'écrit, mais augmentent en calcul », Insee Première, ${ }^{\circ} 1426$.

Jonas N. (2013), « Les capacités des adultes à maîtriser des informations écrites ou chiffrées. Résultats de l'enquête PIAAC 2012 », Insee Première, n 1467.

Jonas N., Lebrère A., Pommier P. et Trosseille B. (2013), « Mesurer les compétences des adultes : comparaison de deux enquêtes ", Insee Analyses, $\mathrm{n}^{\circ} 13$.

Murat F. et Rocher T. (2015), « L'évaluation des compétences des adultes. Quelles contraintes ? Quelles spécificités », Éducation \& Formations, n 86-87, pp. 83-98.

OCDE et Statistique Canada (2000), La littératie à l'ère de l'information. Rapport final de l'enquête internationale sur la littératie des adultes, Paris, OCDE.

OCDE (2013), OECD Skills Outlook 2013. First Results from the Survey of Adult Skills, Paris, OCDE.

OCDE (2016), Les clés de l'intégration. Les réfugiés et autres groupes nécessitant une protection, Paris, OCDE.

Trosseille B. et Rocher T. (2015), « Les évaluations standardisées des élèves : perspective historique », Éducation \& Formations, ${ }^{\circ}$ 86-87, pp. 15-35.

Vallet L.-A., Bonnet G., Émin J.-C., Levasseur J., Rocher T., Blum A., Guérin-Pace F., Vrignaud P., d'Haultfœuille X., Murat F., Verger D. et Zamora P. (2002), « Enquête méthodologique Information et Vie Quotidienne - Tome 1 : bilan du test 1, novembre $2002 »$, Document de travail, série Méthodologie statistique, $\mathrm{n}^{\circ} \mathrm{C} 0202$, Insee. 
